Colloquium Rauricum Band 2

Zweihundert Jahre Homer-Forschung

Rückblick und Ausblick 


\title{
Colloquia Raurica
}

\author{
Herausgegeben von \\ Jakob Frey-Clavel, Joachim Latacz, \\ Hansjörg Reinau, Jürgen von Ungern-Sternberg
}

Die Colloquia Raurica behandeln aktuelle allgemein interessierende wissenschaftliche Fragestellungen, deren Schwerpunkt das Altertum mit seiner Wirkungsgeschichte bis zur Gegenwart bildet. Zur Erzielung möglichst vielseitig abgestützter wissenschaftlicher Erkenntnisse erörtern die eingeladenen Vertreter der thematisch betroffenen geisteswissenschaftlichen Disziplinen das Tagungsthema im gemeinsamen Gespräch. Die Ergebnisse werden in der Folge publiziert. Die Colloquia Raurica finden in der Römerstiftung Dr. René Clavel auf dem Landgut Castelen in Augusta Raurica bei Basel statt. Sie werden in zweijährigem Turnus abgehalten. 
Colloquium Rauricum Band 2

\section{Zweihundert Jahre Homer-Forschung}

Rückblick und Ausblick

Herausgegeben von

Joachim Latacz

\section{宙}

B. G. Teubner Stuttgart und Leipzig 1991 


\section{Gedruckt mit Unterstützung}

von Herrn und Frau

Dr. Jakob und Antoinette Frey-Clavel, Basel

Die Deutsche Bibliothek - CIP-Einheitsaufnahme

\section{Zweihundert Jahre Homer-Forschung}

Rückblick und Ausblick

Hrsg. von Joachim Latacz. - Stuttgart ; Leipzig : Teubner, 1991

(Colloquium Rauricum Bd. 2)

ISBN 3-519-07412-5

NE: Latacz, Joachim [Hrsg.];

Colloquium Rauricum: Colloquium Rauricum

Das Werk einschließlich aller seiner Teile ist urheberrechtlich geschützt. Jede Verwertung außerhalb der engen Grenzen des Urheberrechtsgesetzes ist ohne Zustimmung des Verlages unzulässig und strafbar.

Das gilt besonders für Vervielfältigungen, Übersetzungen,

Mikroverfilmungen und die Einspeicherung und Verarbeitung in elektronischen Systemen.

(C) B.G. Teubner Stuttgart 1991

Printed in Germany

Gesamtherstellung: Passavia Druckerei GmbH Passau 